\title{
Responses re: concerns from Kim et al. about our article, "Energy use, blue water footprint, and greenhouse gas emissions for current food consumption patterns and dietary recommendations in the US"
}

\author{
Michelle S. Tom ${ }^{1}$ (D) Paul S. Fischbeck ${ }^{2,3}$ - Chris T. Hendrickson ${ }^{1,2}$
}

Published online: 16 February 2016

(C) Springer Science+Business Media New York 2016

\section{Dear Editor,}

I am writing in response to the thoughtful letter from Kim, Nachman, Neff, and Spiker (hereafter, Kim et al.), who themselves wrote in response to our article, "Energy Use, Blue Water Footprint, and Greenhouse Gas Emissions for Current Food Consumption Patterns and Dietary Recommendations in the US," which was recently published in Environment Systems and Decisions. We agree with points raised by Kim et al., particularly that the media coverage has, at times, mischaracterized our findings and that the suggestion that meat consumption may be an environmentally sustainable food choice is not a good or helpful one. In this response, we want to direct readers toward the news articles that most accurately portrayed our results and to clarify the actual findings of our article, which do not simply favor meat consumption over fruits and vegetables.

First, let us respond to the points about the news coverage of our work. Indeed, some of the news media has erroneously extrapolated from our findings and claimed that vegetarianism is damaging to the environment. Instead, the relevant point that should be taken away from the CMU press release is that environmental impacts vary

Michelle S. Tom

mtom@andrew.cmu.edu

1 Department of Civil and Environmental Engineering, Carnegie Mellon University, 5000 Forbes Ave, Pittsburgh, PA 15213, USA

2 Departments of Engineering and Public Policy, Carnegie Mellon University, 5000 Forbes Ave, Pittsburgh, PA 15213, USA

3 Department of Social and Decision Sciences, Carnegie Mellon University, 5000 Forbes Ave, Pittsburgh, PA 15213, USA between individual foods within the same food group. Not all meats are equal nor are all fruits and vegetables equal with regard to energy use, blue water footprint, and GHG emissions. Our results do not analyze vegetarian diets, and we have made efforts to clarify our findings in subsequent interactions with the press. These subsequent interactions contributed to the production of several excellent pieces that provided accurate and nuanced summary and analysis of our work. In particular, we would like to direct readers toward articles written by the Washington Post (Whoriskey 2015), the Huffington Post (Hanson 2015), and Vice Magazine (Verger 2015).

Our analysis did not compare vegetarian to non-vegetarian diets. Rather, we compared the current US diet to the USDA recommended diet, which includes meats, abet at lower levels than are currently consumed. Our findings indicate that a shift to the USDA recommended diet would result in greater levels of energy use, blue water footprint, and GHG emissions through the US food supply system. These findings are largely driven by the recommended increase in consumption of fruits, vegetables, and dairy products, which would accompany a recommended decrease in consumption of sugars, fats, and oils. While our results do not focus on vegetarian diets, they do indicate that there is considerable variation in environmental resource use and emissions among non-meat foods. Dairy, fruits, and vegetables, for example, are more emissions intensive per calorie than sugars, fats, and oils. Thus, increasing consumption of dairy, fruits, and vegetables at the expense of sugars, fats, and oils will have negative implications for the environment.

In closing, we again thank Kim et al. for their letter and agree with their comments. We hope that this letter has helped to steer readers toward the most accurate media coverage and helped to clarify our findings. Finally, we 
want to emphasize that further work must be done on the relationship between diet and the environment going forward, and we look forward to contributing to that important and growing body of research.

\section{References}

Hanson H (2015) A study did not actually find that vegetarianism hurts the planet. Huffington Post. http://www.huffingtonpost.com/entry/ vegetarian-bad-for-environment-debunked_567072d7e4b0e29215 0f95a4

Verger R (2015) No, bacon isn't better for the environment than lettuce. Vice. https://news.vice.com/article/no-bacon-isnt-betterfor-the-environment-than-lettuce

Whoriskey $P$ (2015) Is a vegetarian diet really better for the environment? Science takes aim at the conventional wisdom. Washington Post. https://www.washingtonpost.com/news/wonk/ wp/2015/12/18/being-a-vegetarian-might-make-you-feel-environ mentally-superior-why-that-may-be-wrong/ 